\title{
Propiedades del Eichhornia crassipes (Jacinto de agua), Schoenoplectus colifornicus (Junco), y el Phragmites australis (Carricillo)
}

\author{
Properties of Eichhornia crassipes (Jacinto de agua), Schoenoplectus \\ colifornicus (Junco), and Phragmites australis (Carricillo)
}

\author{
Alejandro Mena Ayala ${ }^{1}$ \\ Recibido: 13/01/2020 - Aprobado: 20/12/2020 - Publicado: 18/06/2021
}

\begin{abstract}
RESUMEN
El presente trabajo de investigación ha servido para determinar la capacidad de remoción de metales pesados y encontrar el vegetal adecuado, aprovechando las bondades de las siguientes especies: Eichhornia crassipes (Jacinto de agua, flor de boca o camalote), Schoenoplectus colifornicus (Junco o totora), y Phragmites australis (Carricillo o gramínea trepadora), para ello se construyeron tres estanques de madera debidamente impermeabilizados de las siguientes dimensiones: 0.30 metros de ancho $\times 0.40$ metros de alto y 1.60 metros de largo, haciendo un volumen de 0.192 metros cúbicos, ubicada próximo a una vivienda en el centro poblado de Nueva Esperanza en el distrito de Santa María provincia de Huaura, región Lima.

En cada estanque se sembraron las tres variedades de plantas y a cada una de ellas ha ingresado agua residual de la vivienda, de manera ininterrumpida y luego de más de 90 días de su crecimiento se contrataron los servicios de un laboratorio acreditado Bureau Veritas para realizar los análisis respectivos. Dichos resultados indican que los tres tipos de vegetales asimilan los metales elegidos, siendo el más eficiente el Eichhornia Crassipes (Jacinto de agua), con $94.73 \%$ para el Niquel, $84.36 \%$ para el Plomo, y $78.48 \%$ para el cadmio, en el caso del Schoenoplectus Colifornicus (Junco), para el Niquel resultó $83.00 \%$, Plomo $61.23 \%$ y Cadmio $51.90 \%$, y para el Phragmites Australis (carricillo), en el caso del Niquel fue $79.90 \%$, Plomo $60.23 \%$ y Cadmio $29.11 \%$, por lo tanto se recomienda el Jacinto de agua para captar metales pesados de aguas residuales domésticas y/o industriales.

Mientras que en el cuadro de análisis respecto a los tres tipos de vegetales, en el primer caso el resultado de los tres tipos de vegetales que crecen en forma silvestre en los pantanos cercanos a la zona, formando grandes comunidades, mientras que el segundo análisis corresponde a los tres tipos de vegetales que han sido sembrados en los estanque en los cuales ingresa agua residual domestica de una vivienda, precisamente para notar las diferencias en cuanto al contenido de metales pesados, siendo los resultados los siguientes: $57.00 \%$ para el Jacinto de agua, $42.00 \%$ para el junco y $37.00 \%$ para el carricillo, con ello se demuestra que de los tres tipos de vegetales estudiados el Jacinto de agua es el más eficiente, por lo tanto es recomendable para captación de metales pesados de las aguas residuales domésticas.
\end{abstract}

Palabra clave: Eichhornia crassipes (Jacinto de agua); Schoenoplectus colifornicus (Junco); y el Phragmites australis (carricillo); como depurador de metales pesados.

\begin{abstract}
This research work has served to determine the ability to remove heavy metals and find the appropriate plant, taking advantage of the benefits of the following species: Eichhornia crassipes (Water hyacinth, mouth flower or camalote), Schoenoplectus colifornicus (Junco or totora), and Phragmites australis (Carricillo or climbing grass), for this, three duly waterproofed wooden ponds of the following dimensions were built: 0.30 meters wide $x 0.40$ meters high and 1.60 meters long, making a volume of 0.192 cubic meters, located next to a house in the town of Nueva Esperanza in the district of Santa María, Huaura province, Lima region.

The three varieties of plants were planted in each pond and each one of them has entered residual water from the house, uninterruptedly and after more than 90 days of growth, the services of an accredited Bureau Veritas laboratory were hired to perform the analyzes respective. These results indicate that the three types of vegetables assimilate the chosen metals, the most efficient being Eichhornia Crassipes (water hyacinth), with $94.73 \%$ for Nickel, $84.36 \%$ for Lead, and $78.48 \%$ for cadmium, in the case of Schoenoplectus Colifornicus (Junco), for Nickel it was $83.00 \%$, Lead $61.23 \%$ and Cadmium 51.90\%, and for Phragmites Australis (carricillo), in the case of Nickel it was 79.90\%, Lead $60.23 \%$ and Cadmium $29.11 \%$, so Therefore, water hyacinth is recommended to capture heavy metals from domestic and / or industrial wastewater.
\end{abstract}

1 Ingeniero de Minas, Doctorando en Ciencias Ambientales. E-mail: alexmenaayala@hotmail.com - ORCID: https://orcid.org/0000-0001-5906-8390 
While in the analysis table regarding the three types of vegetables, in the first case the result of the three types of vegetables that grow wild in the swamps near the area, forming large communities, while the second analysis corresponds to the three types of vegetables that have been planted in the ponds in which the domestic residual water of a house enters, precisely to notice the differences in terms of the content of heavy metals, the results being the following: $57.00 \%$ for the water hyacinth , $42.00 \%$ for the reed and $37.00 \%$ for the reed, with this it is shown that of the three types of vegetables studied, the water hyacinth is the most efficient, therefore it is recommended for capturing heavy metals from domestic wastewater.

Keyword: Eichhornia Crassipes (Jacinto de agua); Schoenoplectus Colifornicus (Junco); and Phragmites Australis (carricillo); as a heavy metal scrubber.

\section{INTRODUCCION}

El Jacinto de agua o lechuguin (Eichhornia crassipes), es una planta acuática originaria de la cuenca del Amazonas, también de otros cuerpos de agua de esta región, así como de lagos y pantanos del oeste del Brasil (Barrett \& Forno, 1982). Se le considera planta ornamental y flota libremente en la superficie del agua debido a que sus hojas se inflan como globos, lo que les favorece para flotar; actualmente se encuentra distribuido entre el trópico y subtrópico, entre los $39^{\circ} \mathrm{N}$ y $39^{\circ} \mathrm{S}$ (Guevara Granja, María Fernanda; Ramírez Cando, 2015).

Poma Llantoy \& Valderrama Negrón (2014), desarrollaron una investigación, en laboratorio, para evaluar las propiedades fitorremediadoras del Jacinto de agua. Evaluaron la capacidad de sorción de los iones metálicos Cd (II) y Hg (II), concluyendo que esta especie es "potencialmente aprovechable para remover cadmio (II) y mercurio (II) en aguas contaminadas".

Sandoval Vilchez (2019) estudió la eficiencia del Jacinto de agua y de la lenteja de agua, con metales pesados (cadmio). Concluyó "que Eichhornia crassipes presenta una eficiencia de $83.57 \%$ mientras que Lemna minor $L$. presenta eficiencia de $39.35 \%$ ".

Quispe Baldeón et al. (2017) estudiaron la eficiencia del Jacinto de agua en la remoción de aguas residuales en una laguna experimental de la Universidad La Unión (Lima, Perú). Determinaron 31\% de eficiencia para plomo total.

Así mismo, Jaramillo \& Flores (2012), utilizaron Lemna minor (lenteja de agua) y Eichhornia crassipes (Jacinto de agua) en aguas residuales de la actividad minera, considerándolas como bioacumuladores de sales. Concluyeron que la capacidad de absorción de las macrófitas acuáticas al mercurio fue del 29,5\%, resaltando su eficiencia para remover este elemento del agua.

El Schoenoplectus americanus, conocido comúnmente con el nombre de junco, especie vegetal muy frecuente en los humedales de la costa peruana, es una especie que pertenece a la familia de las Cioeráceas; por su abundancia y los servicios ecológicos que brinda es de gran importancia ecológica (Aponte, H. \& Cano, 2007; Aponte Ubillús, 2007).

Aponte Ubillús (2007) reporta que el "junco se adapta en estanques poco profundos a veces como plantas acuáticas flotantes y otras sumergidas"; agrega que este vegetal recibe nutrientes minerales da partir del agua que ingresa al estanque, que propicia su uso en wetlands.
Bedoya Escobar (2014) reportó que el Schoenoplectus californicus (junco) evidencia "actividad fitorremediadora en agua contaminada con arsénico", a nivel de laboratorio. Resalta, además, su capacidad de tolerar altas concentraciones de arsénico.

El Phragmites australis (carricillo), es una planta que se desarrolla tanto en aguas dulces como salobres, su presencia indicadora alto nivel freático y por ello contribuye a identificar fisonómicamente las zonas húmedas. Crece por debajo de los 1600 metros de altitud (León et al., 1995).

Amabilis-Sosa et al. (2016), determinaron, en un estudio experimental, la factibilidad técnica de utilizar Phragmites australis en humedales artificiales para el tratamiento de aguas residuales con contenido de mercurio. El carrizo es utilizado desde tiempos muy antiguos para las esteras, actualmente su uso se ha visto disminuido en las viviendas populares, sin embargo, del oficio de hacer esteras vive mucha gente alrededor del mundo.

Calcular cual es la capacidad depuradora de los carrizales no es tema fácil, porque intervienen diferentes factores. Esta capacidad depuradora depende, por ejemplo, de la época del año relacionada con la actividad vegetativa, o de la cantidad de nutrientes presentes en el suelo. (Colmenarejo, F, Cirujano, S., Rubio, A., García, Mª 1999).

\section{MÉTODOS}

Para el presente trabajo de investigación se eligió la localidad de Nueva Esperanza, Paraje Pampa de Animas Baja, ubicada en el distrito de Santa María, provincia de Huaura y región Lima. Se prepararon tres estanques de madera de las siguientes dimensiones 0.40 metros de alto x 0.30 metros de ancho x 1.60 metros de largo, haciendo un volumen de 0.192 metros cúbicos de capacidad; en el primer estanque, el Eichhornia Crassipes (Jacinto de agua, flor de boca o camalote); en el segundo estanque, el Schoenoplectus Colifornicus (Junco o totora), y, en el tercer estanque, el Phragmites Australis (Carricillo y gramínea trepadora).

Para el caso del junco y carricillo se sembró de la siguiente manera: se colocaron agregados por un espesor aproximadamente de 5 centímetros, en seguida compost y tierra negra por un espesor aproximado de 10 centímetros y en seguida se sembró los juncos y carricillos pequeños o tiernos, para luego verter agua negra de los residuos de una vivienda, previo a ello se instaló con tuberías y válvulas de control, como se puede apreciar en la Figura 1. 
Para sembrar el Jacinto de agua solo se necesitaba el estanque, el cual se llenó con agua residual y se sembró con la especie indicada, porque estas plantas son flotantes y no necesitan de sustratos, sus raíces están suspendidas en el agua.

Para la instalación de tuberías se siguió el mismo procedimiento para los tres estanques, la instalación se hizo a los bordes de una pequeña acequia por el cual eventualmente escurre agua, se hizo una instalación con una tubería de plástico de 1 pulgada de diámetro y el agua que ingresaba es producto de las aguas residuales de una vivienda elegida, donde habitan 4 personas, sin embargo el agua durante el día ingresa muy poco porque las personas salen al trabajo y las aguas residuales ingresan con mayor volumen es por la noches y mañanas.

Una vez que se sembraron los tres tipos de vegetales se colocó un cerco en la zona, para evitar el ingreso de animales y/o personal extraño. Durante el crecimiento de las plantas se realizó un control para el ingreso proporcional de las aguas residuales a los tres estanques. (Ver Figura 2).

En la Figura 3, se aprecia las plantas tiernas, después de haber sido sembradas:

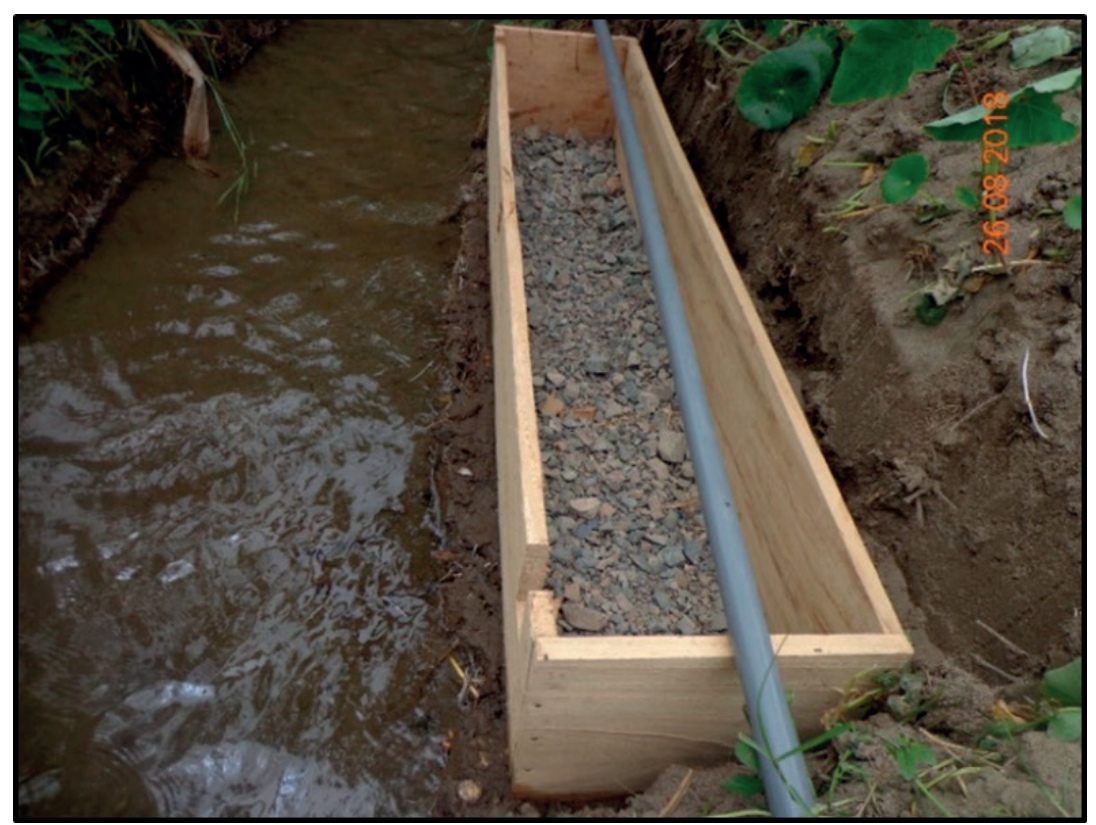

Figura 1. Estanque de madera con base de agregados calcáreos

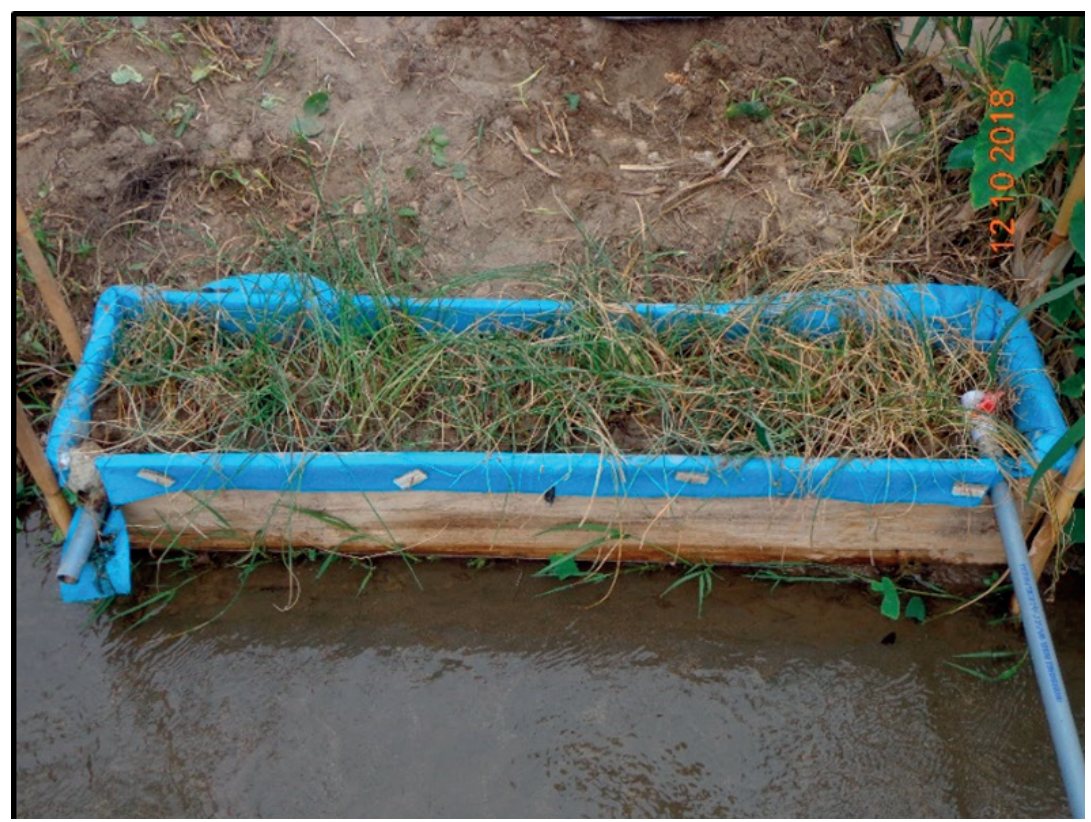

Figura 2. Estanque de madera con plantaciones del Junco 
En la Figura 4, se puede apreciar los tres estanques con los tres tipos de vegetales después de haber sido sembradas, cabe reiterar que la zona donde se instalaron los recipientes se ha tenido que proteger mediante cercos preservar las condiciones de los experimentos.

De acuerdo a la leyenda interna (Tablas 3, 4 y 5), A.W.3, significa que la muestra del tallo del Jacinto de agua ha sido tomado del estanque sembrado para el experimento, mientras que el punto D.W.3, significa la muestra del Jacinto de agua ha sido tomado de un pantano natural cerca de la zona, para su prueba de comparación en ambas condiciones, es decir la primera muestra ha sido tomada del estanque sembrado, mientras que la segunda muestra ha sido de un pantano natural, con fines experimentales, para medir la capacidad de retención de metales pesados, de cada especie.

\section{RESULTADOS}

Luego de 90 días de haber sido sembrado los tres tipos se tomaron muestras de aguas, mediante laboratorio acreditado, tanto al ingreso como a la salida de los tres

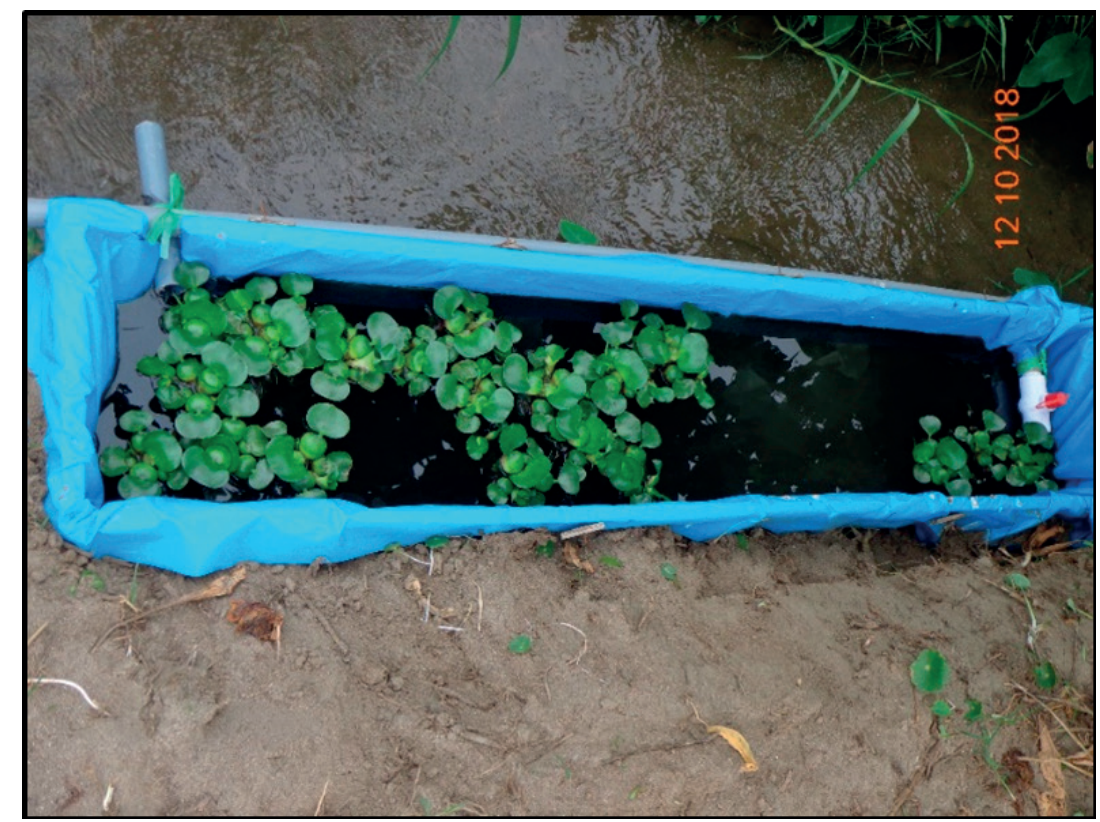

Figura 3. Estanque con agua negra para sembrar el Jacinto de agua

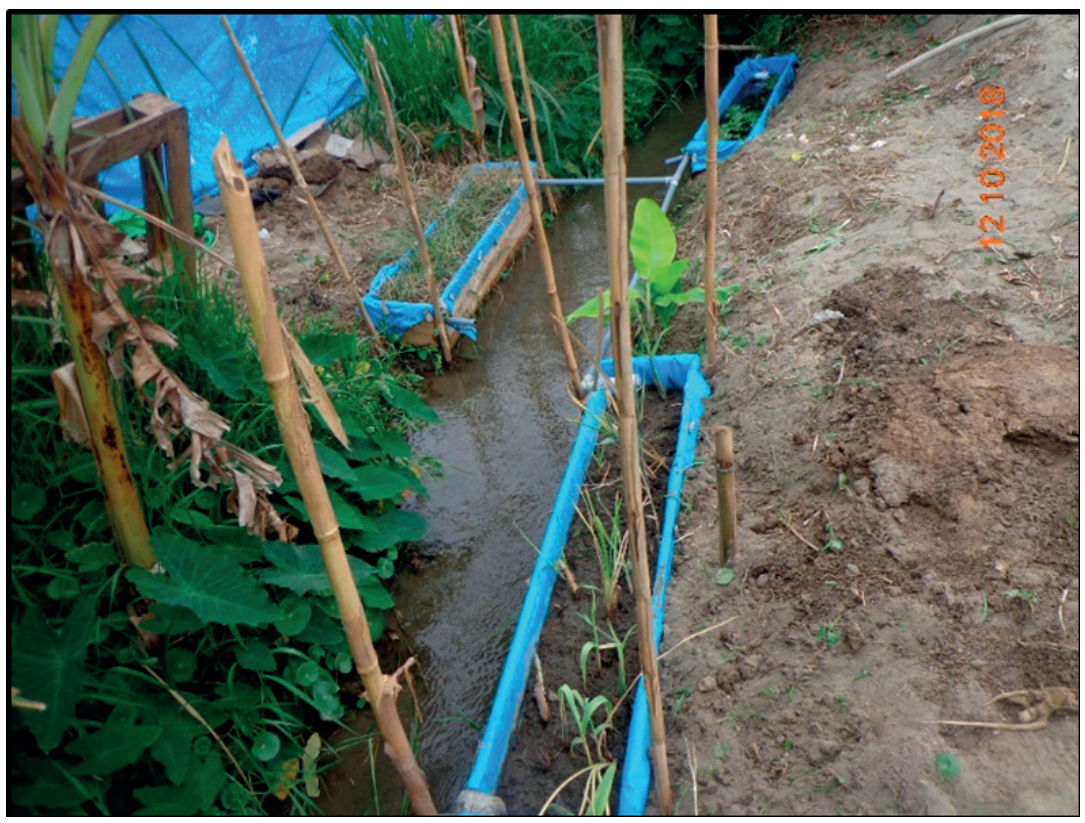

Figura 4. Se observa los tres tipos de plantas sembradas 
estanques. También se tomaron muestras de los tallos tanto de los cultivados en los estanques y otros de las mismas variedades que crecen en bofedales naturales cercanos a la zona. A partir del informe $\mathrm{N}^{\circ} 10578 \mathrm{~L} / 19-\mathrm{MA}$, con fecha del 30 de enero, de dicha entidad, se elaboró la Tabla 1, para el Jacinto de agua.

Los datos de la Tabla 1 ilustran una disminución del contenido del níquel, plomo y cadmio. Con mayor detalle de los promedios, la Tabla 2 muestra el porcentaje de remoción, para el Jacinto de agua y para los metales pesados indicados.
Los datos de la Tabla 3 ilustran una disminución del contenido del níquel, plomo y cadmio, debido a la acción del junco. La tabla 2 muestra el porcentaje de remoción, para esta especie y para los metales pesados indicados.

A continuación, los datos de la Tabla 5 ilustran una disminución del contenido del níquel, plomo y cadmio, debido a la acción del carricillo. Con mayor detalle de los promedios, la Tabla 6 muestra el porcentaje de remoción, para esta especie y para los metales pesados indicados.

Tabla 1. Resumen de contenido de metales pesados en estanque de Jacinto de agua

\begin{tabular}{cccccccccccc}
\hline \multirow{2}{*}{ Metal mg/kg } & \multicolumn{3}{c}{ Antes del ingreso al agua residual (AR) } & \multicolumn{4}{c}{ Después del paso por agua residual } \\
& M1 & M2 & M3 & M4 & M5 & M1 & M2 & M3 & M4 & M5 \\
\hline $\mathrm{Ni}$ (tot) & 0.0500 & 0.0003 & 0.0002 & 0.0003 & 0.0004 & 0.0004 & 0.0009 & 0.0005 & 0.0003 & 0.0006 \\
$\mathrm{~Pb}$ (tot) & 0.0007 & 0.0110 & 0.0016 & 0.0258 & 0.0210 & 0.0021 & 0.0018 & 0.0020 & 0.0018 & 0.0017 \\
$\mathrm{Cd}$ (tot) & 0.0020 & 0.0015 & 0.0014 & 0.0015 & 0.0015 & 0.0002 & 0.0001 & 0.0002 & 0.0005 & 0.0007 \\
\hline
\end{tabular}

Fuente: Elaboración propia a partir del Informe Laboratorio Bureau Veritas

Tabla 2. Porcentaje de remoción del Jacinto de agua para algunos metales pesados

\begin{tabular}{ccccc}
\hline Metal $\mathrm{mg} / \mathrm{kg}$ & $\begin{array}{c}\text { Antes del AR } \\
\text { Promedio }\end{array}$ & $\begin{array}{c}\text { Después del AR } \\
\text { Promedio }\end{array}$ & Resta & \% retención \\
\hline $\mathrm{Ni}$ (tot) & 0.0102 & 0.0005 & 0.0097 & $94.73 \%$ \\
$\mathrm{~Pb}$ (tot) & 0.0120 & 0.0019 & 0.0101 & $84.36 \%$ \\
$\mathrm{Cd}$ (tot) & 0.0016 & 0.0003 & 0.0012 & $78.48 \%$ \\
\hline
\end{tabular}

Fuente: Elaboración propia a partir del Informe Laboratorio Bureau Veritas

Tabla 3. Resumen de contenido de metales pesados en estanque de Junco

\begin{tabular}{cccccccccccc}
\hline \multirow{2}{*}{ Metal mg/kg } & \multicolumn{4}{c}{ Antes del ingreso al agua residual (AR) } & \multicolumn{4}{c}{ Después del paso por agua residual } \\
& M1 & M2 & M3 & M4 & M5 & M1 & M2 & M3 & M4 & M5 \\
\hline $\mathrm{Ni}$ (tot) & 0.0500 & 0.0003 & 0.0002 & 0.0003 & 0.0004 & 0.0022 & 0.0020 & 0.0017 & 0.0015 & 0.0013 \\
$\mathrm{~Pb}$ (tot) & 0.0007 & 0.0110 & 0.0016 & 0.0258 & 0.0210 & 0.0051 & 0.0049 & 0.0046 & 0.0044 & 0.0043 \\
$\mathrm{Cd}$ (tot) & 0.0020 & 0.0015 & 0.0014 & 0.0015 & 0.0015 & 0.0012 & 0.0010 & 0.0008 & 0.0005 & 0.0003 \\
\hline
\end{tabular}

Fuente: Elaboración propia a partir del Informe Laboratorio Bureau Veritas

Tabla 4. Porcentaje de remoción del Junco para algunos metales pesados

\begin{tabular}{ccccc}
\hline Metal mg/kg & $\begin{array}{c}\text { Promedio Antes } \\
\text { del AR }\end{array}$ & $\begin{array}{c}\text { Promedio } \\
\text { Después del AR }\end{array}$ & Resta & $\%$ de retención \\
\hline $\mathrm{Ni}$ (tot) & 0.01024 & 0.0017 & 0.0085 & 83.0 \\
$\mathrm{~Pb}$ (tot) & 0.01202 & 0.0047 & 0.0074 & 61.23 \\
$\mathrm{Cd}$ (tot) & 0.00158 & 0.0008 & 0.0008 & 51.90 \\
\hline
\end{tabular}

Fuente: Elaboración propia a partir del Informe Laboratorio Bureau Veritas

Tabla 5. Resumen de contenido de metales pesados en estanque de Carricillo

\begin{tabular}{cccccccccccc}
\hline \multirow{2}{*}{ Metal $\mathbf{m g} / \mathbf{k g}$} & \multicolumn{3}{c}{ Antes del ingreso al agua residual (AR) } & \multicolumn{5}{c}{ Después del ingreso al agua residual (AR) } \\
& M1 & M2 & M3 & M4 & M5 & M1 & M2 & M3 & M4 & M5 \\
\hline $\mathrm{Ni}$ (tot) & 0.0500 & 0.0003 & 0.0002 & 0.0003 & 0.0004 & 0.0025 & 0.0023 & 0.0022 & 0.0018 & 0.0015 \\
$\mathrm{~Pb}$ (tot) & 0.0007 & 0.0110 & 0.0016 & 0.0258 & 0.0210 & 0.0059 & 0.0056 & 0.0048 & 0.0039 & 0.0037 \\
$\mathrm{Cd}$ (tot) & 0.0020 & 0.0015 & 0.0014 & 0.0015 & 0.0015 & 0.0016 & 0.0012 & 0.0010 & 0.0010 & 0.0008 \\
\hline
\end{tabular}

Fuente: Elaboración propia a partir del Informe Laboratorio Bureau Veritas 
Tabla 6. Porcentaje de remoción del Carricillo para algunos metales pesados

\begin{tabular}{ccccc}
\hline Metal mg/kg & Promedio Antes del AR & Promedio Después del AR & Resta & \% de retención \\
\hline $\mathrm{Ni}$ (tot) & 0.0102 & 0.0021 & 0.0082 & 79.9 \\
$\mathrm{~Pb}$ (tot) & 0.0120 & 0.0048 & 0.0072 & 60.23 \\
$\mathrm{Cd}$ (tot) & 0.0016 & 0.0011 & 0.0005 & 29.11 \\
\hline
\end{tabular}

Fuente: Elaboración propia a partir del Informe Laboratorio Bureau Veritas

Los datos de la Tabla 5 ilustran una disminución del contenido del níquel, plomo y cadmio. Con mayor detalle de los promedios, la tabla 6 muestra el porcentaje de remoción, para el Carricillo y para los metales pesados indicados.

Para evaluar la significancia estadística de los resultados, se aplicó la prueba no paramétrica de Wilcoxon para comparar muestras relacionadas, con nivel de confianza $95 \%$.

Para el Jacinto de agua, de acuerdo con la Tabla 7, presenta una disminución estadísticamente significativa para el metal pesado cadmio $(\mathrm{p}<0.05)$

Tabla 7. Prueba de Wilcoxon: Retención de metales pesados por Jacinto de agua

\begin{tabular}{cccc}
\hline Metal & Diferencia & $\mathbf{Z}$ & Valor $\mathbf{p}$ \\
\hline Niquel & 0.0082 & -0.365 & 0.715 \\
Plomo & 0.0072 & -1.214 & 0.225 \\
Cadmio & 0.0005 & -2.023 & 0.043 \\
\hline
\end{tabular}

Fuente: Elaboración propia

Por otro lado, de acuerdo con la Tabla 8, el Junco presenta una disminución estadísticamente significativa para el metal pesado cadmio $(\mathrm{p}<0.05)$

Tabla 8. Prueba de Wilcoxon: Retención de metales pesados por Junco

\begin{tabular}{cccc}
\hline Metal & Diferencia & $\mathbf{Z}$ & Valor $\mathbf{p}$ \\
\hline Niquel & 0.0082 & -0.674 & 0.715 \\
Plomo & 0.0072 & -1.254 & 0.125 \\
Cadmio & 0.0005 & -2.073 & 0.033 \\
\hline
\end{tabular}

Fuente: Elaboración propia

Para el Jacinto de agua, de acuerdo con la Tabla 7, presenta una disminución estadísticamente significativa para el metal pesado cadmio $(p<0.05)$ (Ver Tabla 9).

Tabla 9. Prueba de Wilcoxon: Retención de metales pesados por Carricillo

\begin{tabular}{cccc}
\hline Metal & Diferencia & $\mathbf{Z}$ & Valor $\mathbf{p}$ \\
\hline Niquel & 0.0082 & -0.677 & 0.498 \\
Plomo & 0.0072 & -1.204 & 0.225 \\
Cadmio & 0.0005 & -2.032 & 0.042 \\
\hline
\end{tabular}

Fuente: Elaboración propia

Además, con el objetivo de conocer y tener un comparativo se tomaron muestras de los tres tipos de tallos vegetales, los que han crecido durante varios meses con aguas residuales domesticas del vertimiento de una vivienda y otras tres muestras que han sido tomadas de tallos vegetales de las tres variedades de unas bofedales cerca de la zona, es decir del Jacinto de agua, junco y carricillo respectivamente, los resultados finales se notan en el informe $\mathrm{N}^{\circ}$ 10579L/19-MA. De la tabla 10.

De los resultados obtenidos se puede deducir que el Jacinto de agua es una especie vegetal que retiene entre sus rizomas y tallos los siguientes metales: Aluminio, Titanio, Manganeso, Cobalto, Cobre, Zinc, Selenio, Bario, Uranio, Manganeso, Calcio y Fierro, hasta un $57 \%$ en promedio entre todos los metales en comparación al grupo de vegetal que crece en los humedales naturales (Ver Tabla 11).

De los resultados obtenidos se puede deducir que el carricillo es una especie vegetal que retiene entre sus rizomas y tallos los siguientes metales: Aluminio, Fósforo, Titanio, Zinc, Selenio, Estroncio, Molibdeno, Sodio, Potasio, Calcio y Fierro, hasta un porcentaje del $37 \%$ en promedio entre todos los metales en comparación al grupo de vegetales que crecen en los humedales naturales (Ver Tabla 12).

\section{DISCUSIÓN}

Para los metales pesados níquel y plomo, la acción del Jacinto de agua, junco y carricillo, se muestra una acción de remoción, la misma que no es significativa (valor $\mathrm{p}>0.05$ ). En cambio, para el cadmio, su capacidad de remoción es significativa para las tres especies. En mayor porcentaje de remoción lo presenta el Jacinto de agua (78\%) frente al $51 \%$ (Junco) y 29\% del carricillo, para el cadmio.

Los resultados del trabajo de campo han permitido ratificar las propiedades biorremediadoras del Jacinto de agua, al igual que Barrett \& Forno (1982), con mayor eficiencia que las otras dos especies analizadas. Se coincide con Aponte Ubillús (2007) respecto al desenvolvimiento del junco en la biorremediación de metales pesados. A diferencia de Sandoval Vilchez (2019), $(84 \%, 2019)$, el porcentaje de remoción del Jacinto de agua, alcanzó $78 \%$.

La acción del carrizo tiene menor eficiencia que el junco y Jacinto de agua, en la biorremediación de aguas residuales.

\section{CONCLUSIONES}

- Las tres especies estudiadas, presentan capacidad significativa de remoción del cadmio el Eichhornia Crassipes (Jacinto de agua) 78\%, el Schoenoplectus 
Tabla 10. Informe de Ensayo N 10579l/19-Ma, Para Jacinto de Agua. Comparativo del Jacinto de agua en estado natural y del estanque piloto (Wetland)

\begin{tabular}{ccccc}
\hline Metal, $(\mathbf{m g} / \mathbf{k g})$. & Jacinto de agua del estanque artificial & Jacinto de agua en estado natural & Retención & \% Retención \\
\hline $\mathrm{Al}$ & 42.95 & 7.61 & 35.34 & $82 \%$ \\
$\mathrm{Ti}$ & 2.21 & 0.76 & 1.45 & $66 \%$ \\
$\mathrm{Mn}$ & 18.05 & 6.32 & 11.73 & $65 \%$ \\
$\mathrm{Co}$ & 0.08 & 0.03 & 0.05 & $63 \%$ \\
$\mathrm{Cu}$ & 0.64 & 0.28 & 0.36 & $56 \%$ \\
$\mathrm{Zn}$ & 2.32 & 2.28 & 0.04 & $2 \%$ \\
$\mathrm{Se}$ & 0.86 & 0.1 & 0.76 & $88 \%$ \\
$\mathrm{Mo}$ & 0.23 & 0.18 & 0.05 & $22 \%$ \\
$\mathrm{Sn}$ & 0.16 & 0.08 & 0.08 & $50 \%$ \\
$\mathrm{Ba}$ & 1.33 & 0.45 & 0.88 & $66 \%$ \\
$\mathrm{U}$ & 0.33 & 0.03 & 0.3 & $91 \%$ \\
$\mathrm{Mg}$ & 456.67 & 224.85 & 231.82 & $51 \%$ \\
$\mathrm{Ca}$ & 1367.6 & 1045.09 & 322.51 & $24 \%$ \\
$\mathrm{Fe}$ & 110.58 & 36.45 & 74.13 & $67 \%$ \\
Porcentaje general de retención del Jacinto de agua & & & $57 \%$ \\
\hline
\end{tabular}

NOTA: El porcentaje de retención resulta de la siguiente manera, ejemplo para el Mn. 18.05-6.32 = 11.73 Entonces $11.73 \times 100 / 18.08=64.9861$ y redondeando resulta $65 \%$

Tabla 11. Informe de Ensayo N 10579l/19-Ma, para El Junco. Comparativo del junco en estado natural y del estanque piloto (Wetland)

\begin{tabular}{ccccc}
\hline Metal, $(\mathrm{mg} / \mathrm{kg})$. & Junco sembrado en estanque artificial & Junco del pantano natural & Retención & $\%$ Retención \\
\hline $\mathrm{Al}$ & 106.3 & 46.92 & 59.38 & $56 \%$ \\
$\mathrm{P}$ & 384.55 & 378.44 & 6.11 & $2 \%$ \\
$\mathrm{Ti}$ & 5.26 & 3.1 & 2.16 & $41 \%$ \\
$\mathrm{Cr}$ & 0.95 & 0.69 & 0.26 & $27 \%$ \\
$\mathrm{Mn}$ & 151.64 & 68.56 & 83.08 & $55 \%$ \\
$\mathrm{Co}$ & 0.07 & 0.05 & 0.02 & $29 \%$ \\
$\mathrm{Sr}$ & 20.84 & 7.7 & 13.14 & $63 \%$ \\
$\mathrm{Ba}$ & 9.52 & 2.7 & 6.82 & $72 \%$ \\
$\mathrm{Na}$ & 2886.2 & 1851.48 & 1034.72 & $36 \%$ \\
$\mathrm{Ca}$ & 961.64 & 559.21 & 402.43 & $42 \%$ \\
$\mathrm{Fe}$ & 206.25 & 114.43 & 91.82 & $45 \%$
\end{tabular}

Tabla 12. Informe de Ensayo N 10579//19-Ma, para el Carricillo. Comparativo del Carricillo en estado natural y del estanque piloto

\begin{tabular}{ccccc}
\hline Metal $(\mathrm{mg} / \mathrm{kg})$ & Carricillo del estanque sembrado & Carricillo del bofedal natural & Retención & $\%$ Retención \\
\hline $\mathrm{Al}$ & 23.03 & 11.28 & 11.75 & $51 \%$ \\
$\mathrm{P}$ & 441.3 & 405 & 36.3 & $8 \%$ \\
$\mathrm{Ti}$ & 3.11 & 1.1 & 2.01 & $65 \%$ \\
$\mathrm{Zn}$ & 8.89 & 6.85 & 2.04 & $23 \%$ \\
$\mathrm{Se}$ & 0.12 & 0.09 & 0.03 & $25 \%$ \\
$\mathrm{Sr}$ & 6.45 & 3.38 & 3.07 & $48 \%$ \\
$\mathrm{Mo}$ & 0.87 & 0.26 & 0.61 & $70 \%$ \\
$\mathrm{Na}$ & 1904.79 & 1267.05 & 637.74 & $33 \%$ \\
$\mathrm{~K}$ & 7082.28 & 5585.3 & 1496.98 & $21 \%$ \\
$\mathrm{Ca}$ & 942.91 & 764.47 & 178.44 & $19 \%$ \\
$\mathrm{Fe}$ & 115.63 & 64.86 & 50.77 & $44 \%$
\end{tabular}


Colifornicus (Junco), 51 \% y, en menor proporción, el Phragmites Australis (carricillo), 29 \%. Por lo que se deduce que el Jacinto de agua resultas el mejor depurador de metales pesados.

- Las tres especies mostraron niveles de remoción del níquel y plomo, sin llegar a una significatividad aceptable.

- Los tres vegetales considerados, en el primer caso los que crecen de manera silvestre formando grandes comunidades y los que fueron cultivados en los estanques artificiales, cuyos resultados para el caso de metales pesados fueron: $57 \%$ para el Eichhornia Crassipes (Jacinto de agua), $42 \%$ Schoenoplectus Colifornicus (Junco), y $37 \%$ para el Phragmites Australis (carricillo).

- Para la prueba piloto se debería preparar un estanque de mayor capacidad de tal manera que el tamaño de los tallos de los tres tipos de vegetales pueda alcanzar tamaños normales, puesto que el espacio reducido limita el desarrollo de las raíces.

- Por los resultados obtenidos se puede recomendar que este tipo de experimentos se podría realizar para aguas residuales que tengan más componentes metálicos como las aguas residuales de las industrias.

- Por los resultados obtenidos se puede recomendar que el Eichhornia Crassipes (Jacinto de agua), es el más eficiente en captar metales pesados de las aguas residuales ya sean domésticas o industriales.

- Es necesario continuar con este tipo de experimentos en otras zonas del país, y posteriormente ser aplicables para tratamientos de aguas a niveles industriales o domésticas.

- Finalmente, con respecto al vegetal del junco se deberían tener políticas del estado para su conservación, teniendo en cuenta que del tallo de este vegetal se elaboran varios tipos de artesanías que son el sustento de cientos de peruanos, y también del carricillo.

\section{REFERENCIAS}

Amabilis-Sosa, L. E., Siebe, C., Moeller-Chávez, G., \& DuránDomínguez-De-bazúa, M. del C. (2016). Remoción de mercurio por Phragmites Australis empleada como barrera biológica en humedales artificiales inoculados con cepas tolerantes a metales pesados. Revista Internacional de Contaminacion Ambiental, 32(1), 47-53. http://www.scielo.org.mx/scielo.php?script=sci arttext\&pid=S0188-49992016000100047

Aponte, H. \& Cano, A. (2007). Estudio preliminar de la distribución de Schoenoplectus americanus en el Perú. Libro de Resúmenes de La XVI Reunión Científica Del Instituto de Investigaciones En Ciencias Biológicas Antonio Raymondi. https://museohn.unmsm.edu.pe/docs/pub_dico/ APONTE 2009.pdf
Aponte Ubillús, H. A. (2007). Respuesta de Schoenoplectus americanus (Pers.) Vol. ex Sch. \& R. Séll . (Cyperaceae) " junco " a diferentes concentraciones de nutrientes [Universidad Nacional Mayor de San Marcos]. http:// aplicaciones.cientifica.edu.pe/repositorio/catalogo/ data/25.pdf

Barrett, S. C. H., \& Forno, I. W. (1982). Style morph distribution in new world populations of Eichhornia crassipes (Mart.) Solms-Laubach (water hyacinth). Aquatic Botany, 13(C), 299-306. https://doi.org/10.1016/0304-3770(82)90065-1

Bedoya Escobar, B. M. (2014). Evaluación de la actividad fitorremediadora del Schoenoplectus californicus "junco" en agua contaminada con arsénico. 149. http://repositorio. unjbg.edu.pe/handle/UNJBG/2029

Colmenarejo, F, Cirujano, S., Rubio, A., García, Ma , E. J. (1999). Diagnóstico de la calidad de las aguas del Parque Natural El Hondo. Actas de Las I Jornadas Científicas Sobre El Parque Natural El Hondo. https://www.researchgate. net/publication/293486022_Actas_de_las_I_Jornadas Cientificas_sobre_el_Parque_Natural_El_Hondo

Guevara Granja, María Fernanda; Ramírez Cando, L. J. (2015). Eichhornia crassipes, su invasividad y potencial fitorremediador. La Granja. Revista de Ciencias de La Vida, 22(2), 5-11. https://doi.org/10.17163/lgr.n22.2015.01

Jaramillo, M. D. S., \& Flores, E. (2012). Fitorremediación mediante el uso de dos especies vegetales lemna minor (Lenteja de agua) y Echhornia crassipes (Jacinto de agua) en aguas residuales producto de la actividad minera. [Universidad Politécnica Salesiana]. In Universidad Politécnica Saleciana. https://dspace.ups.edu.ec/ handle/123456789/2939

León, B., Cano, A., \& Young, K. (1995). La Flora Vascular de los Pantanos de Villa, Lima, Perú : Adiciones y guía para las especies comunes. Museo de Historia Natural UNMSM, May 1995, 1-39. https://museohn.unmsm.edu.pe/docs/pub botanica/Publicaciones Botánica Serie B No 38.pdf

Poma Llantoy, V. R., \& Valderrama Negrón, A. C. (2014). Estudio de los parámetros fisicoquímicos para la fitorremediación de cadmio (II) y mercurio (II) con la especie Eichhornia crassipes (Jacinto de Agua). Revista de La Sociedad Química Del Perú, 80(3), 164-173. https://doi.org/10.37761/rsqp. v80i3.224

Quispe Baldeón, L., Arias Chavez, J. B., Martinez Suarez, C. F., \& Cruz Huaranga, M. (2017). Eficiencia de la especie macrófita Eichhornia crassipes (Jacinto de agua) para la remoción de parámetros fisicoquímicos, metal pesado $(\mathrm{Pb})$ y la evaluación de su crecimiento en función al tiempo y adopción al medio en una laguna experimental. Revista de Investigación Ciencia, Tecnología y Desarrollo, 3(1). https://doi.org/10.17162/rictd.v3i1.650

Sandoval Vilchez, D. J. (2019). EFICIENCIA DEL JACINTO DE AGUA EICHHORNIA CRASSIPES Y LENTEJA DE AGUA LEMNA MINOR L. EN LA REMOCIÓN DE CADMIO EN AGUAS RESIDUALES. In Univerdad Nacional Federico Villareal. http://repositorio.unfv.edu.pe/ handle/UNFV/3256 